\title{
Adaptive Fuzzy Sliding Mode Control of a Pressure-Controlled Artificial Ventilator
}

\author{
Ibrahim M. Mehedi $\mathbb{D}^{1,2}$ Heidir S. M. Shah, ${ }^{1}$ Ubaid M. Al-Saggaf $\left(\mathbb{D},{ }^{1,2}\right.$ Rachid Mansouri $\left(\mathbb{D},{ }^{3}\right.$ \\ and Maamar Bettayeb $\mathbb{D}^{4}$ \\ ${ }^{1}$ Department of Electrical and Computer Engineering (ECE), King Abdulaziz University, Jeddah 21589, Saudi Arabia \\ ${ }^{2}$ Center of Excellence in Intelligent Engineering Systems (CEIES), King Abdulaziz University, Jeddah 21589, Saudi Arabia \\ ${ }^{3}$ Laboratoire de Conception et Conduite des Systemes de Production (L2CSP), Tizi-Ouzou 15000, Algeria \\ ${ }^{4}$ Electrical Engineering Department, University of Sharjah, Sharjah, UAE
}

Correspondence should be addressed to Ibrahim M. Mehedi; imehedi@kau.edu.sa

Received 31 May 2021; Revised 8 June 2021; Accepted 12 June 2021; Published 24 June 2021

Academic Editor: Dilbag Singh

Copyright ( $\odot 2021$ Ibrahim M. Mehedi et al. This is an open access article distributed under the Creative Commons Attribution License, which permits unrestricted use, distribution, and reproduction in any medium, provided the original work is properly cited.

\begin{abstract}
This paper presents the application of adaptive fuzzy sliding mode control (AFSMC) for the respiratory system to assist the patients facing difficulty in breathing. The ventilator system consists of a blower-hose-patient system and patient's lung model with nonlinear lung compliance. The AFSMC is based on two components: singleton control action and a discontinuous term. The singleton control action is based on fuzzy logic with adjustable tuning parameters to approximate the perfect feedback linearization control. The switching control law based on the sliding mode principle aims to minimize the estimation error between approximated single fuzzy control action and perfect feedback linearization control. The proposed control strategy manipulated the airway flow delivered by the ventilator such that the peak pressure will remain under critical values in presence of unknown patient-hose-leak parameters and patient breathing effort. The closed-loop stability of AFSMC will be proven in the sense of Lyapunov. For comparative analysis, classical PID and sliding mode controllers are also designed and implemented for mechanical ventilation problems. For performance analysis, numerical simulations were performed on a mechanical ventilator simulator. Simulation results reveal that the proposed controller demonstrates better tracking of targeted airway pressure compared with its counterparts in terms of faster convergence, less overshoot, and small tracking error. Hence, the proposed controller provides useful insight for its application to real-world scenarios.
\end{abstract}

\section{Introduction}

Several new viruses, epidemics, and even pandemics have emerged in the past 20 years. 774 people have been killed by the severe acute respiratory syndrome (SARS) that first emerged in mid-November 2002 in the Guangdong province, China [1]. In 2009, the World Health Organization (WHO) had declared a new global pandemic called H1N1 influenza. Although the confirmed number of deaths by WHO are 18,500, Dawood [2] in his studies estimates the actual number should be between 151,700 and 575,400. The most recent is the Middle East respiratory syndrome (MERS-CoV) in 2012 with 806 associated deaths reported in
December 2018 mostly in Saudi Arabia [3]. Before the pandemic COVID-19 where to date, over 50 million people have been contacted with the disease with over 1 million deaths recorded since its discovery in the Wuhan province, China, in December 2019. The common cause of death for patients who contracted these diseases are acute respiratory distress syndrome (ARDS) where according to a study, 40 percent of critically ill COVID-19 patients developed this respiratory failure [4-7]. In most cases of ARDS, patients will have to be supported with mechanical ventilation to assist or replace their spontaneous breathing [8].

The earliest usage of mechanical ventilators as a device to provide ventilatory assistance can be traced back to the 18th 
century. However, it was only in the 1950s that the first closed-loop system for mechanical ventilation was introduced. During this time, mechanical bellows and valves were used in mechanical ventilators to cycle gas into the lung, while pneumatic components were used to implement simple proportional (P) or proportional integral (PI) controller [9]. Microprocessors were eventually used to implement those controllers, and since then, many types of closed-loop controls have been proposed.

Walter and Leonhardt [10] classify the different levels of automatic control in artificial ventilation into three categories according to the amount of interaction between the patient and medical devices. In class 1 control loops called device-internal control loops and class 2 control loops called patient-oriented control loops, control signals are measured inside the device. The difference between both classes is that the interaction between the patient and device is possible in class 2 , while there are no such features in class 1 control loops. Instead of using physical parameters, a class 3 control loop used physiological parameters as its control variable and for that it is called physiological compensatory control loops. In this paper, a pressure-based ventilation controller under the class 2 category is developed where the control objective is to track a set-point of target airway pressure.

One type of controller that has been used widely in mechanical ventilation is the PID controller. One of its earliest implementation since the introduction of the microprocessor can be found here [11]. This type of controller is however known for its poor performance on a system where its dynamic is not constant, which is the case for mechanical ventilation. The lung compliance and resistance are two parameters in the mechanical ventilation system that are changing according to the lung volumes and are different from patient to patient. Many researchers have proposed improvement to the PID controller in mechanical ventilation, which includes the addition of an adaptive mechanism [12], an optimization technique [13], and automatic tuning of PID gains [14].

The accurate information of the system dynamics is quite essential for designing nonlinear control systems. Model-based controllers $[15,16]$ are employed, which are more transparent as they are developed from the mathematical models derived from physiological processes. A slight disadvantage could be in obtaining the precise mathematical model required for a good performance especially as patient dynamics are uncertain, which is the same case as inverse dynamic controller [17]. Other types of controller implemented in mechanical ventilation from our literature searches are model predictive control [18], variable-gain control [19], and repetitive control [20].

There are nonlinear control strategies available in the literature that does not require exact knowledge of the system dynamics [21, 22]. Among these controllers, sliding mode control (SMC) is a powerful method with inherited robust attitudes to control complex dynamical systems [23, 24]. However, conventional SMC may potentially experience chattering problems in the control command, which degrades the control performance by exciting unmodeled high-frequency dynamics, which may lead to instability [25]. Noninteger control, or fractional-order control (FOC), has received significant attention in control engineering due to its ability to tune time-varying systems while remaining controllable $[26,27]$. Noninteger calculus significantly improves the performance of PID controllers. The fact has been investigated in previous studies [26-29].

In literature, fuzzy logic control (FLC), which falls under the category of intelligent control, has been widely used as an alternative to control the nonlinear system in presence of unknown dynamics and external perturbations [30, 31]. There were also attempts of using FLC in mechanical ventilation $[32,33]$, which is easy to implement as it does not require explicit mathematical models. However, traditional FLC may require some systematic mechanism for mapping the expert knowledge into the rule base of fuzzy interference system [34, 35]. Hence, the subject control strategy could have limited transparency. Thus, extensive testing with hardware will be required for its validation and verification such that closed-loop stability is guaranteed. To cope with this, it is preferable to augment some adaptive mechanism for adjusting the control parameters automatically while guaranteeing closed-loop stability. In adaptive fuzzy, there are two widely used control approaches: direct and indirect. The former approach is based on adjusting the control parameters with changing dynamics, whereas the later indirect approach is responsible to identify the unknown system parameters [4]. As a result, both of these strategies are striving to achieve the desired system performance by adjusting the fuzzy rules accordingly [36-39].

In literature, various combinations of SMC and FLC have been proposed to handle complex and unmodeled dynamics [40, 41] and have been applied to various nonlinear systems [42-44] including microelectromechanical system (MEMS) resonators [45]. In prior studies [46-48], the authors have implemented fuzzy double hidden layer recurrent neural network to approximate the SMC-based equivalent control, eliminating the unknown disturbance while reducing the impact of switching gain. Similarly, Poursamad and Davaie-Markazi [49] have implemented a variant of hybrid FLC and SMC control titled robust adaptive fuzzy control for Duffing oscillators and atomic force microscopes. The later methodology has been adopted in this article to approximate the ideal control law based on fuzzy approximation theory while handling modeling uncertainties and disturbances through augmentation of SMCbased switching control law.

This paper deals with a design of direct form of adaptive fuzzy sliding mode control (AFSMC) by exploiting the potential of both SMC and FLC strategies to handle complex and unmodeled dynamics. The resultant control expression comprised singleton fuzzy control action, which adjusts itself based on Lyapunov energy function, and switching control element, which aims to minimize the approximation error between fuzzy control and perfect feedback linearization control law. The resultant controller drastically enhances the robustness while guaranteeing the asymptotic stability of the closed-loop system. As a case study, the proposed AFSMC controller is employed for pressure control of the well-known mechanical ventilator system. To visualize the performance attributes, a simulation model of 
the mechanical ventilator system is modeled in Simulink/ MATLAB environment. Numerical simulations reveal that the direct form of AFSMC is quite effective by steering the pressure in the closed vicinity of the desired pressure curve.

The rest of this paper is organized as follows: the detailed mathematical modeling of ventilator system is given in Section 2. The description of AFSMC controller is presented in Section 3 with detailed stability proof in the sense of Lyapunov. Simulation results of AFSMC along with its comparison with classical PID and SMC are depicted in Section 4. Finally, the paper is concluded in Section 6.

\section{Artificial Ventilator Modeling}

In this paper, a blower-hose-patient system model introduced in [50] consists of 3 main components-the blower, the hose, and the patient-is used to represent the respiratory system model as shown in Figure 1. The function of the blower is to generate the desired pressure $p_{o}$ by compressing the atmospheric air, while the hose links the respiratory module to the patient. A single compartmental model presented in [19] is used to represent the patient's lung model. The aim of the controller is to track the airway pressure $p_{a w}$ that is measured by a sensor positioned inside the module so that it follows the target setpoint $p_{t}$. With that, we can describe the error equation as follows:

$$
e=p_{t}-p_{a w}
$$

During the inhalation process, the patient will inhale the air from the blower $\left(Q_{o}\right)$ into the lung $\left(Q_{p}\right)$, then exhale back to the hose. To avoid the patient from taking the exhaled air back in the next cycle, a leak is positioned before the end of the tube to release the exhaled air $\left(Q_{\text {leak }}\right)$. Hence, we can write the patient flow dynamic as follows:

$$
Q_{p}=Q_{o}-Q_{\text {leak }} \text {. }
$$

The airflow is derived from the pressure differences over resistance; thus, in this model, resistance in the hose $\left(R_{h}\right)$, in the leak $\left(R_{\text {leak }}\right)$, and in the patient's lung $\left(R_{l}\right)$ must also be taken into account. We can therefore describe the relationships between blower flow, leak flow, and patient flow with the resistances as follows:

$$
\begin{aligned}
Q_{o} & =\frac{p_{o}-p_{a w}}{R_{h}}, \\
Q_{\text {leak }} & =\frac{p_{a w}}{R_{\text {leak }}}, \\
Q_{p} & =\frac{p_{a w}-p_{l}}{R_{l}} .
\end{aligned}
$$

The lung pressure can be depicted by the following differential equation:

$$
\dot{p}_{l}=\frac{1}{C_{l}} Q_{p}
$$

where $C_{l}$ denotes the lung compliance. Combining equations (3)-(6) results in the following lung dynamics:

$$
\dot{p}_{l}=\frac{p_{a w}-p_{l}}{C_{l} R_{l}} \text {. }
$$

We can derive the formula for airway pressure by substituting equations (3)-(5) into (2) as shown below:

$$
p_{a w}=\frac{\left(1 / R_{l}\right) p_{l}+\left(1 / R_{h} p_{o}\right)}{\left(1 / R_{l}\right)+\left(1 / R_{h}\right)+\left(1 / R_{\text {leak }}\right)} .
$$

The differential equation for the lung dynamic in (6) can now be rewritten by substituting the airway pressure expression in (8) into it as follows:

$$
\dot{p}_{l}=\frac{-\left(\left(1 / R_{h}\right)+\left(1 / R_{l}\right)\right) p_{l}+\left(1 / R_{h}\right) p_{o}}{R_{l} C_{l}\left(\left(1 / R_{l}\right)+\left(1 / R_{h}\right)+\left(1 / R_{\text {leak }}\right)\right)} .
$$

We can represent (5), (7), and (8) in the state-space form as follows:

$$
\begin{array}{r}
\dot{p}_{l}=A_{h} p_{l}+B_{h} p_{o} \\
{\left[\begin{array}{c}
p_{a w} \\
Q_{p}
\end{array}\right]=C_{h} p_{l}+D_{h} p_{o}}
\end{array}
$$

where $p_{o}$ is the input, $\left[p_{a w}, Q_{p}\right]^{T}$ is the output vector, $p_{l}$ is the state, and

$$
\begin{aligned}
& A_{h}=-\frac{\left(1 / R_{h}\right)+\left(1 / R_{\text {leak }}\right)}{R_{l} C_{l}\left(\left(1 / R_{l}\right)+\left(1 / R_{h}\right)+\left(1 / R_{\text {leak }}\right)\right)}, \\
& B_{h}=\frac{\left(1 / R_{h}\right)}{R_{l} C_{l}\left(\left(1 / R_{l}\right)+\left(1 / R_{h}\right)+\left(1 / R_{\text {leak }}\right)\right)}, \\
& C_{h}=\left[\frac{\left(1 / R_{l}\right)}{\left(1 / R_{l}\right)+\left(1 / R_{h}\right)+\left(1 / R_{\text {leak }}\right)}-\frac{\left(1 / R_{h}\right)+\left(1 / R_{l}\right)}{R_{l}\left(\left(1 / R_{l}\right)+\left(1 / R_{h}\right)+\left(1 / R_{\text {leak }}\right)\right)}\right]^{T}, \\
& D_{h}=\left[\frac{\left(1 / R_{h}\right)}{\left(1 / R_{l}\right)+\left(1 / R_{h}\right)+\left(1 / R_{\text {leak }}\right)} \frac{\left(1 / R_{h}\right)}{R_{l}\left(\left(1 / R_{l}\right)+\left(1 / R_{h}\right)+\left(1 / R_{\text {leak }}\right)\right)}\right]^{T} .
\end{aligned}
$$




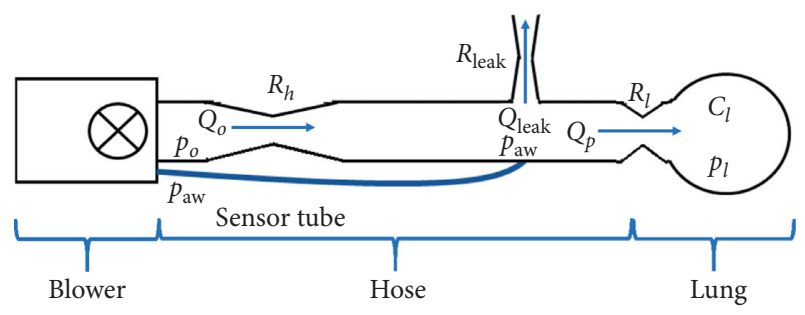

FIgURE 1: Blower-hose-patient system.

The blower system on the other hand can be represented by the following state-space model:

$$
\begin{aligned}
& \dot{x}_{b}=A_{b} x_{b}+B_{b} P_{\text {con }}, \\
& p_{o}=C_{b} x_{b} .
\end{aligned}
$$

Finally, the complete general state-space model of the artificial ventilator is obtained by coupling expression in (10) with (16) and (17) as follows:

$$
\begin{aligned}
\dot{x}_{p} & =\left[\begin{array}{c}
\dot{x}_{b} \\
\dot{p}_{l}
\end{array}\right] \\
& =\left[\begin{array}{cc}
A_{b} & 0 \\
B_{h} C_{b} & A_{h}
\end{array}\right]\left[\begin{array}{c}
x_{b} \\
p_{l}
\end{array}\right]+\left[\begin{array}{c}
B_{b} \\
0
\end{array}\right] P_{\text {con }}
\end{aligned}
$$

\section{Design of AFSMC}

This section presents the direct form of adaptive control employing FLC and SMC to achieve precise tracking performance while ensuring closed-loop stability of the mechanical ventilator. This has been accomplished by augmenting fuzzy approximation theory and principle of sliding mode control theory in the proposed AFSMC method, which proves to be robust against parametric uncertainties and perturbations.

3.1. Problem Statement. Consider the class of $n$th order control-affine nonlinear system given as

$$
\begin{aligned}
\mathbf{x}_{n} & =f(\mathbf{x}, t)+g(\mathbf{x}, t) u+d(t), \\
y & =x,
\end{aligned}
$$

where $f(\mathbf{x}, t)$ and $g(\mathbf{x}, t)$ are the smooth and continuous bounded nonlinear functions, $\mathbf{x} \in R^{n}$ is the state vector assumed to be measurable, $u \in R$ and $y \in R$ are the control input and output of the system, respectively, and $d(t)$ is the unknown but bounded disturbances such that $d(t) \leq D$. If the state transition function $f(\mathbf{x}, t)$ and control input vector $g(\mathbf{x}, t)$ are perfectly known, then a perfect feedback linearization control law to follow the desired state trajectory $x_{d}$ in presence of modeling uncertainties and exogenous disturbances is given by

$$
u^{*}=\frac{1}{g(\mathbf{x}, t)}\left[-f(\mathbf{x}, t)+\ddot{x}_{d}-k_{1} \dot{e}(t)-k_{2} e(t)\right] .
$$

where $e(t)=x-x_{d}$ is the tracking error, while $k_{1}$ and $k_{2}$ are the positive gain constants. However, in practice, it is not possible to determine the exact value of $f(\mathbf{x}, t)$ and $g(\mathbf{x}, t)$; therefore, it is difficult to apply control law given by (20). Hence, it is preferable to introduce some adaptive component in feedback linearization control law that will adjust the control parameters to improve tracking performance and to guarantee closed-loop stability.

\subsection{AFSMC Design Based on Universal Approximation} Theorem. This section presents the design of AFSMC control for pressure control of mechanical ventilator systems. The proposed control methodology integrates the fuzzy approximation theory and principles of sliding mode control into a fuzzy controller, which proves to be insensitive against modeling uncertainties and external perturbations. The decision-making capability of fuzzy control employing linguistic information has been proved to be effective for controlling nonlinear complex systems. The prime objective of the fuzzy controller is to approximate the pressure control command $P_{\text {con }}$ such that it will approach the ideal control law $u^{*}$ given by (20) to arbitrary accuracy by defining multiple output fuzzy sets with singleton membership functions. With a view that the fuzzy rule base grows exponentially as the number of input variables increases, the sliding surface as a function of state variables is considered the input to the fuzzy set. The sliding surface being input to the fuzzy system is defined as

$$
s(t)=\dot{e}(t)+k_{1} e(t)+k_{2} \int e(t) \mathrm{d} t .
$$

where $e(t)=P_{t}-P_{a w}, \lambda$ is the Laplace operator, and the variables $k_{1}, k_{2}$, and $k_{3}$ are the coefficients of Hurwitz polynominal $h(\lambda)=\lambda^{n-1}+k_{n-1} \lambda^{n-2}+\cdots+k_{1}$, such that all the root lies in the open left half-plane. Taking the derivative of the sliding surface given by (21) implies

$$
\dot{s}(t)=\ddot{e}(t)+k_{1} \dot{e}(t)+k_{2} e(t) .
$$

To make the IF part of the input variable $s$, symmetric and uniformly distributed triangular membership functions are used, whereas the output of the fuzzy system $P_{f z}$ is defined as the singleton control action $\alpha_{i}, i=1,2, . ., n$, where $n$ is the number of fuzzy rules as shown in Figure 2.

The $i$ th fuzzy linguistic rule involved the design process is written in the following form.

Rule $i$ : If $s$ is in the domain of $F_{s}^{i}$, then $P_{f z_{i}}$ is $\alpha_{i}$.

The control law $P_{f z}$ is obtained by the center of gravity defuzzification method [45], which implies

$$
P_{f z}(\alpha, \xi)=\frac{\sum_{i=1}^{m} \alpha_{i} \cdot \omega_{i}}{\sum_{i=1}^{m} \omega_{i}},
$$

where $\omega_{i}$ is the firing strength of the $i$ th rule. In order to cope with uncertain and complex unmodeled system dynamics, the singleton control action $\alpha_{i}$ is chosen to be an adjustable parameter with respect to changing environment [34]. The resultant adaptive fuzzy control law $P_{f z}$ as a function of tunable parameter $\alpha$ can explicitly be expressed as 

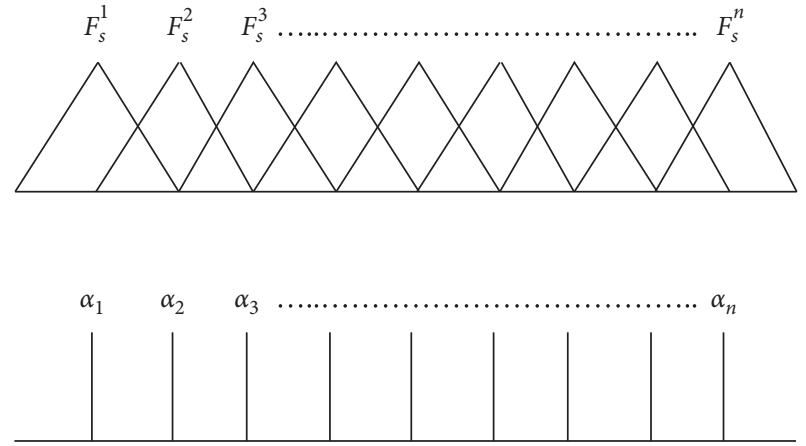

FIGURE 2: Singleton control.

$$
P_{f z}(\alpha, \xi)=\boldsymbol{a}^{T} \xi
$$

where the vector $\alpha=\left[\alpha_{1}, \alpha_{2}, \ldots, \alpha_{m}\right]^{T}$ represents the parameter vector and $\xi=\left[\xi_{1}, \xi_{2}, \ldots, \xi_{m}\right]^{T}$ symbolize the regressive vector, while $\xi_{i}$ is defined as

$$
\xi_{i}=\frac{\omega_{i}}{\sum_{i=1}^{m} \omega_{i}} .
$$

The absolute feedback linearization control based on the universal approximation theorem [18] can be expressed as

$$
\begin{aligned}
P_{\text {con }}^{*} & =P_{f z}^{*}\left(\boldsymbol{\alpha}^{*}, \boldsymbol{\xi}\right)+\epsilon \\
& =\boldsymbol{\alpha}^{* T} \boldsymbol{\xi}+\epsilon,
\end{aligned}
$$

where $\epsilon$ depicts the approximation error described as

$$
\epsilon=P_{\text {con }}^{*}-P_{f z}^{*}\left(\boldsymbol{\alpha}^{*}, \boldsymbol{\xi}\right),
$$

To realize the perfect feedback linearization control $P_{\text {con }}^{*}$, the fuzzy control law $P_{f z}^{*}\left(\alpha^{*}, \xi\right)$ can be approximated as follows:

$$
\widehat{P}_{f z}(\widehat{\mathbf{\alpha}}, \boldsymbol{\xi})=\widehat{\boldsymbol{\alpha}}^{T} \boldsymbol{\xi}
$$

where $\widehat{\alpha}$ is the estimation of $\alpha^{*}$. Despite the capacity that $P_{f z}$ will strive to approach $P_{\text {con }}^{*}$, there is always some residual error between $P_{f z}$ and $P_{\text {con }}^{*}$. This error will be minimized by augmenting the sliding mode-based discontinuous switching term $P_{s w}$ in the control expression described as

$$
P_{\text {con }}=\widehat{P}_{f z}(\widehat{\boldsymbol{\alpha}}, \boldsymbol{\xi})+P_{\text {sw }},
$$

where

$$
P_{s w}=-K \operatorname{sign}(s(t)),
$$

The switching control law is intended to minimize the error between the designed fuzzy law $\widehat{P}_{f z}$ and perfect feedback linearization control law $P_{\text {con }}^{*}$. The resultant control law $P_{\text {con }}$ given by (29) leads to the robust control approach to achieve smooth and precise tracking performance even if the system under consideration subjects to high degree of uncertainty.

Theorem 1. Consider the mechanical ventilator system given by (38), the control law given by (29), with fuzzy control element $\widehat{P}_{f z}$ given by (28) and the switching control element
$P_{s w}$ given by (30), ensures the finite-time stability while tracking error converges to zero.

Proof. To prove the finite-time stability, the expressions of perfect feedback linearization control law given by (20) and sliding mode dynamics given by (22) are solved for $P_{\text {con }}^{*}$, which yields the following expression:

$$
P_{\text {con }}^{*}=\frac{1}{g(\mathbf{x}, t)}\left[g(\mathbf{x}, t) P_{\text {con }}-\dot{s}(t)\right] .
$$

Substituting the control law $P_{\text {con }}$ given by (29) in (31) yields the following sliding mode dynamics:

$$
\dot{s}(t)=g(\mathbf{x}, t)\left[\widehat{P}_{f z}+P_{s w}-P_{\text {con }}^{*}\right] .
$$

Choose the following Lyapunov candidate function:

$$
V_{1}(s(t), \widetilde{\boldsymbol{\alpha}})=\frac{1}{2} s^{2}(t)+\frac{1}{2 \gamma_{1}} \widetilde{\boldsymbol{\alpha}}^{T} \widetilde{\boldsymbol{\alpha}},
$$

where $\gamma_{1}$ is a positive constant. Taking the time derivative of $V_{1}$ yields the following equation:

$$
\begin{aligned}
\dot{V}_{1}(s(t), \widetilde{\boldsymbol{\alpha}}) & =s(t) \dot{\boldsymbol{s}}(t)+\frac{g}{\gamma_{1}} \widetilde{\boldsymbol{\alpha}}^{T} \dot{\tilde{\mathbf{\alpha}}}, \\
& =s(t) g\left(\widehat{P}_{f z}+P_{s w}-P_{\text {con }}^{*}\right)+\frac{g}{\gamma_{1}} \widetilde{\boldsymbol{\alpha}}^{T} \dot{\tilde{\boldsymbol{\alpha}}}, \\
& =s(t) g\left(\widetilde{\boldsymbol{\alpha}}^{T} \xi+P_{s w}-\epsilon\right)+\frac{g}{\gamma_{1}} \widetilde{\boldsymbol{\alpha}}^{T} \dot{\tilde{\boldsymbol{\alpha}}}, \\
& =g \widetilde{\boldsymbol{\alpha}}^{T}\left\{s(t) \xi+\frac{1}{\gamma_{1}} \dot{\tilde{\mathbf{\alpha}}}\right\}+s(t) g\left(P_{s w}-\epsilon\right) .
\end{aligned}
$$

To ensure the finite-time stability of the closed-loop system, let us define the adaptive law as follows:

$$
\dot{\widetilde{\boldsymbol{\alpha}}}=\dot{\widehat{\boldsymbol{\alpha}}}=-\gamma_{1} s(t) \xi
$$

Placing the expression of $P_{s w}$ given by (30) leads to the following expression of $\dot{V}_{1}$ :

$$
\dot{V}_{1}(s(t), \widetilde{\boldsymbol{\alpha}})=-K|s(t)| g-\epsilon s(t) g \leq-K|s(t)| g-|\epsilon s(t)| g
$$

$$
=-(K+|\epsilon|)|s(t)| g \leq 0 .
$$

Considering that the sliding surface $s$ is uniformly continuous, the Lyapunov stability is guaranteed by negative semidefiniteness of $\dot{V}_{1}(s(t), \widetilde{\alpha})$. Asymptotic stability of the closed-loop system follows from Barbalat's lemma [43], such that $(s(t), \widetilde{\alpha}) \longrightarrow(0,0)$ as $t \longrightarrow \infty$.

Proof. The next objective is to find the appropriate value of sliding mode gain $K$, which needs to be smooth enough in order to avoid undesirable chattering phenomenon. If $K$ is made small, it will reduce chattering, but it may lead to inferior tracking performance. Hence, it would be preferable to make the sliding mode gain adaptively to achieve better 
tracking accuracy and closed-loop stability. By replacing $K$ by $\widehat{K}$, the equation of the switching (discontinuous) control law is described as

$$
\widehat{P}_{s w}=-\widehat{K}(t) \operatorname{sign}(s(t)),
$$

where $\widehat{K}$ is the estimation of $K$. Based on this, the control law given by (29) is written as

$$
P_{\text {con }}=\widehat{P}_{f z}(\widehat{\boldsymbol{\alpha}}, \xi)-\widehat{K}(t) \operatorname{sign}(s(t)) .
$$

Theorem 2. Consider the mechanical ventilator system given by (38), the control law given by (42), comprising fuzzy-based controller $\widehat{P}_{f z}$ and switching control element $P_{s w}$, guarantees that the closed-loop system is asymptotically stable in the sense of Lyapunov and the system follows the desired reference output in presence of modeling uncertainties and disturbances.

Proof. Define the estimation error of sliding mode gain as

$$
\widetilde{K}(t)=\widehat{K}(t)-K \text {. }
$$

Now, consider the Lyapunov energy function $V_{2}$ as

$$
V_{2}(s(t), \widetilde{\boldsymbol{\alpha}}, \widetilde{K})=\frac{1}{2} s^{2}(t)+\frac{1}{2 \gamma_{1}} g \widetilde{\boldsymbol{\alpha}}^{T} \widetilde{\boldsymbol{\alpha}}+\frac{1}{2 \gamma_{2}} \widetilde{K}^{2},
$$

where $\gamma_{2}$ is the positive gain constant. The time derivative of $V_{s}$ along the error trajectory given by (44) is

$$
\begin{aligned}
\dot{V}_{2}(s(t), \widetilde{\boldsymbol{\alpha}}, \widetilde{K})= & s(t) \dot{s}(t)+\frac{g}{\gamma_{1}} \widetilde{\boldsymbol{\alpha}}^{T} \dot{\tilde{\boldsymbol{\alpha}}}++\frac{g}{\gamma_{2}} \widetilde{K} \dot{\tilde{K}} \\
= & g \widetilde{\boldsymbol{\alpha}}^{T}\left(s(t) \xi+\frac{1}{\gamma_{1}} \dot{\widehat{\boldsymbol{\alpha}}}^{T}\right)+s(t) g\left(P_{s w}-\epsilon\right) \\
& +\frac{g}{\gamma_{2}} \widetilde{K} \dot{\tilde{K}} \\
= & -\widehat{K}(t)|s(t)| g-\epsilon s(t) g \\
& +\frac{g}{\gamma_{2}}(\widehat{K}(t)-K) \dot{\hat{K}}(t) .
\end{aligned}
$$

To establish asymptotic convergence of the tracking error such that $\dot{V}_{2} \leq 0$, the estimation law is defined as

$$
\dot{\hat{K}}(t)=\gamma_{2}|s(t)|,
$$

By substituting the expression of $\dot{\hat{K}}$, the time derivative of $V_{2}$ given by (45) can be written as

$$
\dot{V}_{2}(s(t), \widetilde{\boldsymbol{\alpha}}, \widetilde{K})=-\widehat{K}(t)|s(t)| g-\epsilon s(t) g+(\widehat{K}(t)-K)|s(t)| g,
$$

$$
=\epsilon s(t) g-K|s(t)| g \leq|\epsilon s(t)| g-K|s(t)| g,
$$

$$
=(K-|\epsilon|)|s(t)| g \leq 0 .
$$

Negative semidefiniteness of $\dot{V}_{2}(s(t), \widetilde{\alpha}, \widetilde{K})$ implies the Lyapunov stability of the system equilibrium $(s(t), \widetilde{\alpha}, \widetilde{K})=(0,0,0)$. Asymptotic stability follows from Barbalat's lemma, see, e.g., [43].

The AFSMC structure given by (42) encompassing fuzzy and switching control elements to track the targeted pressure commands is illustrated in Figure 3.

\section{Numerical Simulations}

The practical applicability of AFSMC for pressure control in the simulated mechanical ventilation system is demonstrated in this section. The nominal values of the system parameters of artificial mechanical ventilator are chosen as $R_{\text {Lung }}=0.005 \mathrm{mbar} / \mathrm{mL} / \mathrm{s}, \quad R_{\text {Leak }}=0.06 \mathrm{mbar} / \mathrm{mL} / \mathrm{s}$, and $R_{\text {hose }}=0.0045 \mathrm{mbar} / \mathrm{mL} / \mathrm{s}$. For computer simulation, the targeted airway pressure is set to be $0.30 \mathrm{mbar}$. Three control methodologies-AFSMC, SMC, and PID—are implemented in order to illustrate the performance comparison. The tradeoff between faster convergence toward the desired values, settling time, and overshoot in the response curves of three control strategies is shown in Figure 4.

The superiority of AFSMC over conventional PID and SMC is clearly observed through simulation results. The AFSMC demonstrates smooth tracking performance with small tracking error compared with its counterparts. Furthermore, the key performance indices of AFSMC, SMC, and PIC control schemes in response to the square wave input of targeted airway pressure are listed in Table 1. It is apparent that no overshoot has been observed in all the simulation cases.

To quantify the tracking performance, the time histories of the magnitude of residual error of the control methodologies under consideration are illustrated in Figure 5, which reveals that the AFSMC scheme adequately follows the desired pressure curve.

\section{Robust Analysis}

In this section, to gain a qualitative insight of the robustness characteristic and sensitivity of AGDI control, a realistic scenario is exercised by introducing $20 \%$ random variations in the parameters of artificial ventilator system such as $R_{l}, R_{h}$, and $R_{\text {leak }}$. The magnitude of ventilator parameter lung compliance $C_{\text {lung, }}$, which determines the change in lung volume per change in trans-pulmonary pressure, is also varied as its value is not constant and changes accordingly with respect to the lung's volume. Computer simulation is performed by commanding the targeted airway pressure of 0.30 mbar for the artificial ventilator. The response curves of targeted and achieved airway pressure are depicted in Figure 6, which reveals effective tracking of pressure commands along with faster convergence toward the reference value. The corresponding control input command is shown in Figure 7 , 


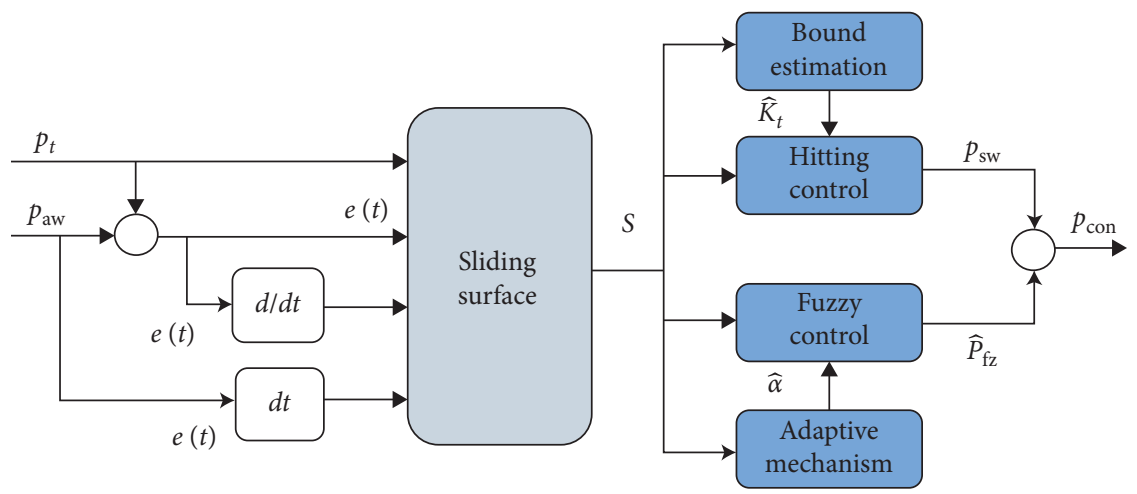

Figure 3: AFSMC block diagram.

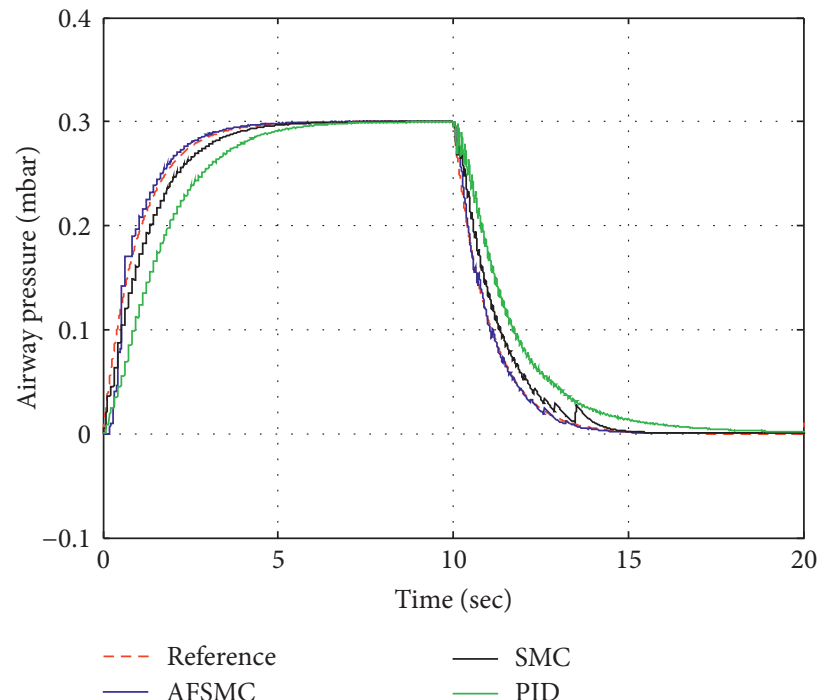

Figure 4: Airway pressure.

TABle 1: Performance comparison.

\begin{tabular}{lccr}
\hline Parameters & AFSMC & SMC & PID \\
\hline Rise time, sec & 2.129 & 2.729 & 3.530 \\
Settling time, $\sec$ & 3.640 & 4.357 & 5.522 \\
\hline
\end{tabular}

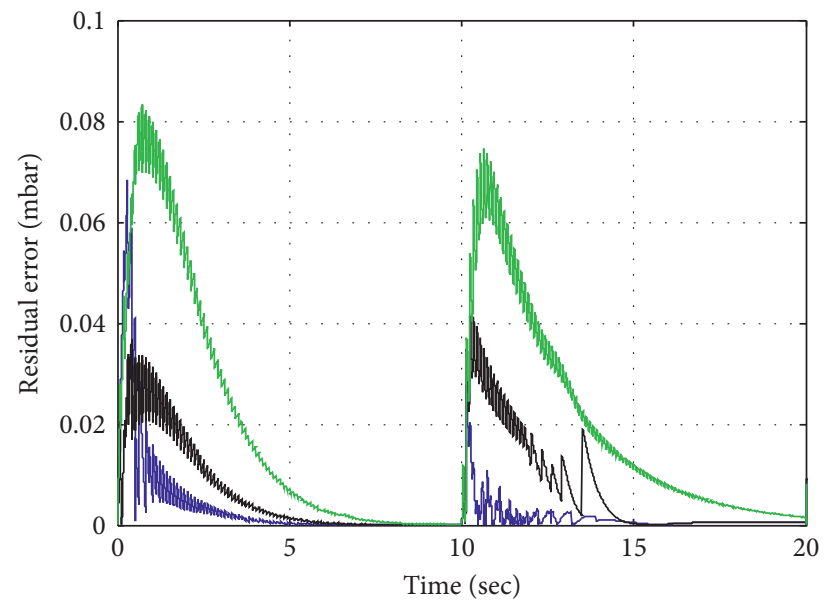

AFSMC

FIgURe 5: Residual error. 


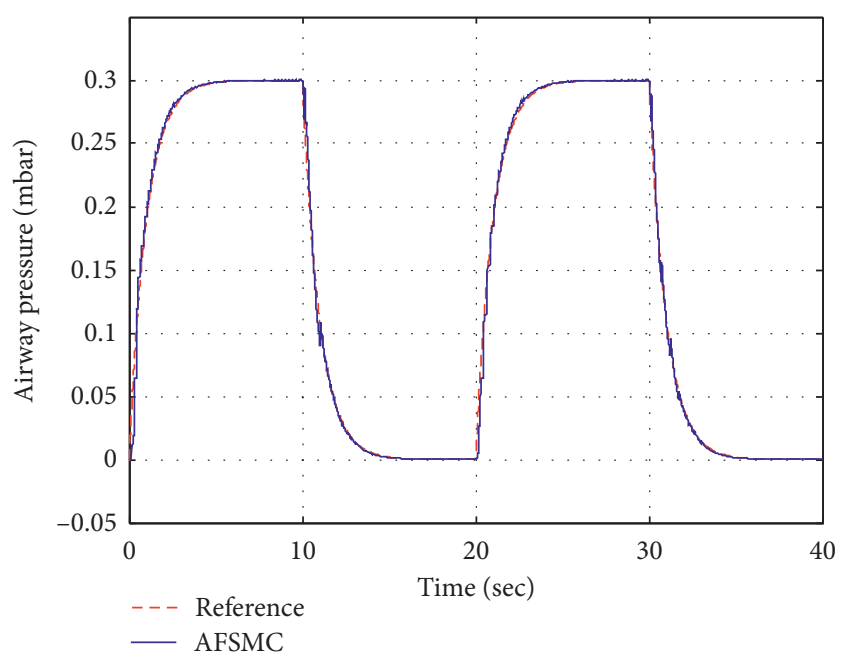

Figure 6: Airway pressure.

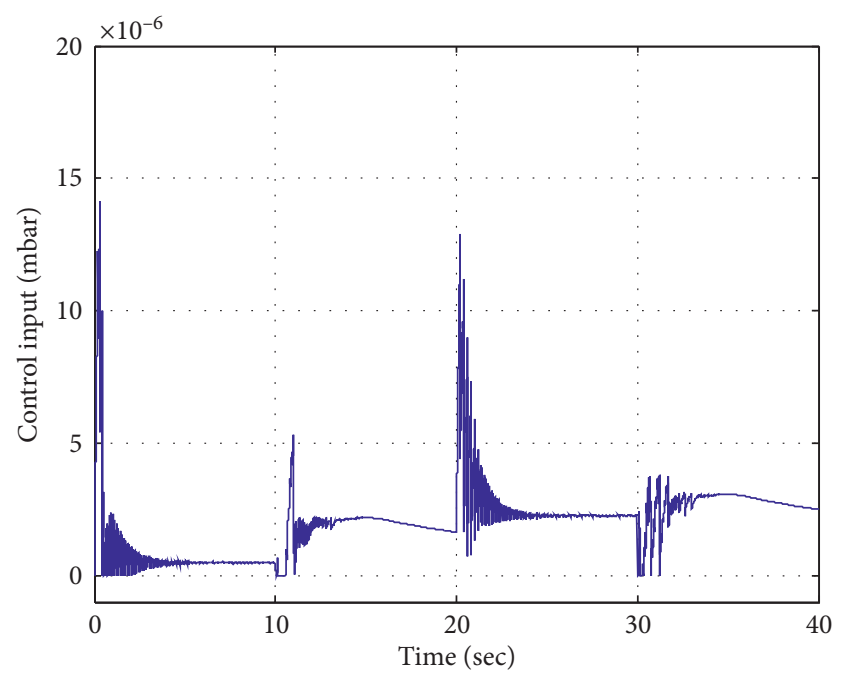

Figure 7: Control input.

which is very much realizable. From simulation results, it is concluded that the AGDI control is made adaptive and robust against parametric variations and proven to be effective and feasible for its applications to real-world systems.

\section{Conclusion}

The paper presents the design of AFSMC for smooth tracking of targeted pressure curves of the mechanical ventilation systems. For performance evaluation, along with AFSMC, two other control approaches (PID and SMC) are also implemented and their performances have been verified in terms of convergence toward the targeted pressure curves of the artificially ventilated human respiratory system. The AFSMC approach reveals better pressure tracking performance by adjusting the ventilator output pressure curves adaptively in comparison with SMC and PID. In future work, the presented approach can further be checked and verified through numerical simulation as well as experimental investigations for a more complex human lung artificially ventilated system.

\section{Data Availability}

No data were used to support this study.

\section{Conflicts of Interest}

The authors declare that they have no conflicts of interest.

\section{Acknowledgments}

This project was funded by the Deanship of Scientific Research (DSR) at King Abdulaziz University, Jeddah, under grant no. GCV19-3-1441. The authors, therefore, acknowledge with thanks DSR for technical and financial support.

\section{References}

[1] M. Chan-Yeung and R.-H. Xu, "SARS: epidemiology," Respirology, vol. 8, pp. S9-S14, 2003.

[2] S. D. Fatimah, A. D. Iuliano, C. Reed et al., "Estimated global mortality associated with the first 12 months of 2009 pandemic influenza a h1n1 virus circulation: a modelling study," The Lancet Infectious Diseases, vol. 12, no. 9, pp. 687-695, 2012.

[3] World Health Organization, Middle East Respiratory Syndrome Coronavirus (Mers-cov), World Health Organization, Geneva, Switzerland, 2019.

[4] S. Ghosh, P. Shivakumara, P. Roy, and T. Lu, "Graphology based handwritten character analysis for human behaviour identification," CAAI Transactions on Intelligence Technology, vol. 5, no. 1, pp. 55-65, 2019.

[5] M. Kaur, V. Kumar, V. Yadav et al., "Metaheuristic-based deep COVID-19 screening model from chest x-ray images," Journal of Healthcare Engineering, vol. 2021, 9 pages, Article ID 8829829, 2021.

[6] D. Singh, V. Kumar, V. Yadav, and M. Kaur, "Deep neural network-based screening model for COVID-19-infected patients using chest x-ray images," International Journal of Pattern Recognition and Artificial Intelligence, vol. 35, no. 3, Article ID 2151004, 2021.

[7] C. Wu, X. Chen, Y. Cai et al., "Risk factors associated with acute respiratory distress syndrome and death in patients with COVID-19 pneumonia in wuhan, China," JAMA Internal Medicine, vol. 180, no. 7, 1031 pages, 2020.

[8] D. Singh, V. Kumar, and M. Kaur, "Densely connected convolutional networks-based COVID-19 screening model," Applied Intelligence, vol. 51, no. 3, pp. 3044-3051, 2021.

[9] M. Borrello, "Modeling and control of systems for critical care ventilation," in Proceedings of the 2005, American Control Conference, pp. 2166-2180, IEEE, Portland, OR, USA, June 2005.

[10] M. Walter and S. Leonhardt, "Control applications in artificial ventilation," in Proceedings of the 2007 Mediterranean Conference on Control \& Automation, pp. 1-6, IEEE, Athens, Greece, June 2007.

[11] K. B. Ohlson, D. R. Westenskow, and W. S. Jordan, "A microprocessor based feedback controller for mechanical ventilation," Annals of Biomedical Engineering, vol. 10, no. 1, pp. 35-48, 1982. 
[12] P. Shi, N. Wang, F. Xie, and H. Su, "Self-adjusting ventilator control strategy based on pid," 2020.

[13] Y. Xu, L. Li, Y. Jia, and Y. Luo, "An optimized controller for bi-level positive airway pressure ventilator," in Proceedings of the 2014 International Conference on Future Computer and Communication Engineering (ICFCCE 2014), March 2014.

[14] D. Acharya and D. K. Das, "Swarm optimization approach to design pid controller for artificially ventilated human respiratory system," Computer Methods and Programs in Biomedicine, vol. 198, Article ID 105776, 2020.

[15] E. P. Martinoni, C. A. Pfister, K. S. Stadler et al., "Model-based control of mechanical ventilation: design and clinical validation," British Journal of Anaesthesia, vol. 92, no. 6, pp. 800-807, 2004.

[16] M. Scheel, T. Schauer, A. Berndt, and O. Simanski, "Modelbased control approach for a CPAP-device considering patient's breathing effort," IFAC-PapersOnLine, vol. 50, no. 1, pp. 9948-9953, 2017.

[17] S. Korrapati and J. -Shiou Yang, "Adaptive inverse dynamics controlz for a two-compartment respiratory system," in Proceedings of the 2016 IEEE International Conference on Consumer Electronics-Taiwan (ICCE-TW), pp. 1-2, IEEE, Nantou, Taiwan, May 2016.

[18] H. Li and W. M. Haddad, "Model predictive control for a multicompartment respiratory system," IEEE Transactions on Control Systems Technology, vol. 21, no. 5, pp. 1988-1995, 2012.

[19] B. Hunnekens, S. Kamps, and N. Van De Wouw, "Variablegain control for respiratory systems," IEEE Transactions on Control Systems Technology, vol. 28, no. 1, pp. 163-171, 2018.

[20] J. Reinders, R. Verkade, B. Hunnekens, N. V. D. Wouw, and O. Tom, "Improving mechanical ventilation for patient care through repetitive control," IFAC-Papers OnLine, vol. 53, 2020.

[21] Y. Pan and J. Wang, "Model predictive control of unknown nonlinear dynamical systems based on recurrent neural networks," IEEE Transactions on Industrial Electronics, vol. 59, no. 8, pp. 3089-3101, 2011.

[22] H. Huaguang Zhang, L. Lili Cui, X. Xin Zhang, and Y. Yanhong Luo, "Data-driven robust approximate optimal tracking control for unknown general nonlinear systems using adaptive dynamic programming method," IEEE Transactions on Neural Networks, vol. 22, no. 12, pp. 22262236, 2011.

[23] K. Khalil Hassan and J. W. Grizzle, Nonlinear Systems, Vol. 3, Prentice-Hall, Upper Saddle River, NJ, USA, 2002.

[24] E,S. Jean-Jacques and W. Li, Applied Nonlinear Control, vol. 199, Prentice-Hall, Englewood Cliffs, NJ, USA, 1991.

[25] A. Abrishamifar, A. Ahmad, and M. Mohamadian, "Fixed switching frequency sliding mode control for single-phase unipolar inverters," IEEE Transactions on Power Electronics, vol. 27, no. 5, pp. 2507-2514, 2011.

[26] U. M. Al-Saggaf, I. M. Mehedi, R. Mansouri, and M. Bettayeb, "State feedback with fractional integral control design based on the Bode's ideal transfer function," International Journal of Systems Science, vol. 47, no. 1, pp. 149-161, 2016.

[27] U. M. Al-Saggaf, I. M. Mehedi, Rachid Mansouri, and Maamar Bettayeb, "Rotary flexible joint control by fractional order controllers," International Journal of Control, Automation and Systems, vol. 15, pp. 134-140, 2017.

[28] M. Ibrahim, "Mehedi. State feedback based fractional order control scheme for linear servo cart system," Journal of Vibroengineering, vol. 20, pp. 782-792, 2018.
[29] I. M. Mehedi and U. M. Al-Saggaf, "Two degrees of freedom fractional controller design: application to the ball and beam system," Measurement, vol. 135, pp. 13-22, 2019.

[30] Ya-C. Hsu and H. A. Malki, "Fuzzy variable structure control for mimo systems,"vol. 1, pp. 280-285, in Proceedings of the 1998 IEEE International Conference on Fuzzy Systems Proceedings. IEEE World Congress on Computational Intelligence (Cat. No. 98CH36228), vol. 1, IEEE, Anchorage, AK, USA, May 1998.

[31] T. Takagi and M. Sugeno, "Fuzzy identification of systems and its applications to modeling and control," IEEE transactions on systems, man, and cybernetics, vol. 15, no. 1, pp. 116-132, 1985.

[32] H. Guler and F. Ata, "Design of a fuzzy-labview-based mechanical ventilator," Computer Systems Science and Engineering, vol. 29, no. 3, pp. 219-229, 2014.

[33] J. Schäublin, M. Derighetti, P. Feigenwinter, S. Petersen-Felix, and A. M. Zbinden, "Fuzzy logic control of mechanical ventilation during anaesthesia," British Journal of Anaesthesia, vol. 77, no. 5, pp. 636-641, 1996.

[34] S. Kundu and D. R. Parhi, "Reactive navigation of underwater mobile robot using anfis approach in a manifold manner," International Journal of Automation and Computing, vol. 14, no. 3, pp. 307-320, 2017.

[35] D. Pelusi, "Optimization of a fuzzy logic controller using genetic algorithms,"vol. 2, pp. 143-146, in Proceedings of the 2011 Third International Conference on Intelligent HumanMachine Systems and Cybernetics, vol. 2, IEEE, Hangzhou, China, August 2011.

[36] Y. Li, H. Wang, B. Zhao, and K. Liu, "Adaptive fuzzy sliding mode control for the probe soft landing on the asteroids with weak gravitational field," Mathematical Problems in Engineering, vol. 2015, Article ID 582948, 8 pages, 2015.

[37] M. Roopaei, M. Zolghadri, and S. Meshksar, "Enhanced adaptive fuzzy sliding mode control for uncertain nonlinear systems," Communications in Nonlinear Science and $\mathrm{Nu}$ merical Simulation, vol. 14, no. 9-10, pp. 3670-3681, 2009.

[38] S. Ali, Hew. Wooi Ping, M. N. Uddin, and K. Salloum Gaeid, "Adaptive fuzzy sliding-mode control into chattering-free im drive," IEEE Transactions on Industry Applications, vol. 51, no. 1, pp. 692-701, 2014.

[39] Li X. Wang and H. Ying, "Adaptive fuzzy systems and control: design and stability analysis," Journal Of Intelligent and Fuzzy Systems-applications in Engineering and Technology, vol. 3, no. 2, 187 pages, 1995.

[40] A. Ishigame, T. Furukawa, S. Kawamoto, and T. Taniguchi, "Sliding mode controller design based on fuzzy inference for nonlinear systems (power systems)," IEEE Transactions on Industrial Electronics, vol. 40, no. 1, pp. 64-70, 1993.

[41] M. Roopaei and M. Zolghadri Jahromi, "Chattering-free fuzzy sliding mode control in mimo uncertain systems," Nonlinear Analysis: Theory, Methods \& Applications, vol. 71, no. 10, pp. 4430-4437, 2009.

[42] C. Otto and P. Hušek, "Adaptive fuzzy sliding mode control for electro-hydraulic servo mechanism," Expert Systems with Applications, vol. 39, no. 11, Article ID 10269, 2012.

[43] F.-J. Lin and S.-L. Chiu, "Adaptive fuzzy sliding-mode control for pm synchronous servo motor drives," IEE Proceedings Control Theory and Applications, vol. 145, no. 1, pp. 63-72, 1998.

[44] S. Liu and L. Ding, "Application of adaptive fuzzy sliding mode controller in pmsm servo system,"vol. 2, pp. 95-98, in Proceedings of the 2010 International Conference on 
Computing, Control and Industrial Engineering, vol. 2, , IEEE, Wuhan, China, June 2010.

[45] S. H. Hossein and A. H. D. Markazi, "Chaos prediction and control in mems resonators," Communications in Nonlinear Science and Numerical Simulation, vol. 15, no. 10, pp. 30913099, 2010.

[46] J. Fei and Y. Chen, "Fuzzy double hidden layer recurrent neural terminal sliding mode control of single-phase active power filter," IEEE Transactions on Fuzzy Systems, vol. 12, 1 page, 2020.

[47] J. Fei, Y. Chen, L. Liu, and Y. Fang, "Fuzzy multiple hidden layer recurrent neural control of nonlinear system using terminal sliding-mode controller," IEEE Transactions on Cybernetics, vol. 12, pp. 1-16, 2021.

[48] J. Fei, Z. Wang, X. Liang, Z. Feng, and Y. Xue, "Fractional sliding mode control for micro gyroscope based on multilayer recurrent fuzzy neural network," IEEE Transactions on Fuzzy Systems, vol. 91 page, 2021.

[49] A. Poursamad and A. H. Davaie-Markazi, "Robust adaptive fuzzy control of unknown chaotic systems," Applied Soft Computing, vol. 9, no. 3, pp. 970-976, 2009.

[50] J. H. T. Bates, Lung Mechanics: An Inverse Modeling Approach, Cambridge University Press, Cambridge, UK, 2009. 